\title{
In Situ Tarsal Metatarsal Dowel Arthrodesis with Trephine Reamers and Cancellous Bone Plugs: A Technique Guide and Case Report
}

\author{
Michael W Downey*, Justin J Fleming and Benjamin Elgamil
}

Aria 3B Orthopaedics, Philadelphia PA, USA

\begin{abstract}
Tarsalmetatarsal (TMT) osteoarthritis is encountered frequently. Pathology at the TMT joints play a role in continued foot fatigue and further compensation of gait laterally to avoid discomfort over the medial and central columns of the midfoot. Sequeula and further pathology to the central TMT joints are multifactorial. Recalcitrant pain, subtle instability, and arthrosis are commonly observed. Patients who fail nonoperative treatment are subjective to reconstructive surgical treatment. We present a straightforward stepwise technique for an in situ arthrodesis of the central TMT joints. We focus on the principles of the dowel arthrodesis with trephine reamers, cancellous bone plugs, and compressive locking plate fixation for a reproducible corrective midfoot fusion.
\end{abstract}

Keywords: Midfoot fusion; In situ midfoot arthrodesis; Dowel midfoot arthrodesis; Allograft plugs for midfoot fusion

\begin{abstract}
Abbreviations: TMT: Tarsalmetatarsal; mm: Millimeters; MRI: Magnetic Resonance Imaging; CAM: Controlled Ankle Motion; mmHG: Millimeters of Mercury, cm: Centimeter, BMA: Bone Marrow Aspirate; VAS: Visual Analogue Scale
\end{abstract}

\section{Introduction}

Tarsalmetatarsal (TMT) osteoarthritis is encountered frequently in a surgeons practice. Recalcitrant pain, subtle instability, and arthrosis are commonly observed rather than positional deformity. Patients who fail nonoperative treatment (to include physical therapy, cortisone injections, orthotic accommodation) are subjective to reconstructive surgical goals to establish a plantigrade, painfree, and functional gait [1]. There have been several techniques described for TMT joint arthritis and degenerative changes [2]. We present a straightforward stepwise technique for an in situ arthrodesis of the central TMT joints.

We focus on the principles of the dowel arthrodesis with trephine reamers, cancellous bone plugs, and compressive locking plate fixation for a reproducible corrective midfoot fusion without sacrificing adjacent anatomical structures and without expansive dissection needs.

\section{Anatomical considerations}

The TMT complex consists of osseous, cartilaginous, capsular and ligamentous configurations of the intertarsal, intermetatarsal, and tarsalmetatarsal architecture [3]. The medial, central, and lateral columns make up an arch and truss type configuration in combination with the long and short plantar ligaments [3]. The columns are independent, with each having fibrous membranes lining the synovial capsule [4]. The central column is formed by the second and third metatarsals and the intermediate and lateral cuneiforms respectively. Specifically, there is limitation on the amount of plantarflexion and dorsiflexion of the second due to the wedge shape of the metatarsal bases and the recession of the second metatarsals between the cuneiforms [5]. The central column is inherently stable and acts as the keystone of the midfoot with only $0.6 \mathrm{~mm}$ of sagittal plane motion $[4,6]$. There is additional function and stability in the frontal plane with increased reliability on the surrounding soft tissues [7]. The articular surfaces are larger dorsally than plantarly and the average sizes for the second and third TMT joints are recorded at $26.9 \mathrm{~mm}$ and $23.6 \mathrm{~mm}$ respectively $[4,8]$. The ligaments of the TMT joints have prudent variability and make up the thickenings of the synovial capsules with longitudinal and oblique fibers [4]. The dorsal ligaments average close to six to eight ligaments per column and are typically flat and conjoined, whereas the plantar ligaments average five ligaments total across all columns and are stronger than their dorsal counterpart [4]. Because of the resistance and stability of the plantar ligaments, soft tissue pathology occurs dorsally [4]. Interosseous ligaments provide increased stability and strength across each column of the TMT joints with the Lisfranc ligament being the most notable ligament that spans the medial aspect of the second metatarsal and the lateral aspect of the medial cunieform [4]. Intermetatarsal ligaments offer further stability to the TMT joints; however the absence of the ligament between the first and second metatarsal predispose this area to injury [9]. Peicha et al. [5] reported in an anatomic study that there is a predisposition of injury for patients that have a shallower depth in the mortise between the medial and lateral cuneiform bones [5].

\section{Surgical technique}

Informed consent was been obtained from the patient and/ or appropriate persons for publication, including any necessary photographs. A healthy, active 45 year-old female presented to clinic with a one year worsening history of midfoot pain. She sustained an isolated TMT joint sprain that was subsequently treated conservatively prior to our encounter with 4-6 weeks of non-steroidal antiinflammatory medications, ice, and elevation.

Radiographs of the left foot (Figure 1) demonstrated a mild subtle osteophyte on the dorsal aspect of second TMT joint but otherwise unremarkable for any further osseous or chondrogenic pathology. An MRI (Figure 2) of the left foot was obtained which revealed the presence

*Corresponding author: Michael W Downey, DPM, AACFAS, Fellow Foot and Ankle, Aria 3B Orthopaedics, 3110 Grant Ave, Philadelphia PA 19114, USA, Tel: 215-464-6600; E-mail: michael.w.downey@gmail.com

Received May 04, 2016; Accepted May 16, 2016; Published May 25, 2016

Citation: Downey MW, Fleming JJ, Elgamil B (2016) In Situ Tarsal Metatarsal Dowel Arthrodesis with Trephine Reamers and Cancellous Bone Plugs: A Technique Guide and Case Report. J Arthritis 5: 198. doi:10.4172/2167-7921.1000198

Copyright: (c) 2016 Downey MW, et al. This is an open-access article distributed under the terms of the Creative Commons Attribution License, which permits unrestricted use, distribution, and reproduction in any medium, provided the original author and source are credited. 
Citation: Downey MW, Fleming JJ, Elgamil B (2016) In Situ Tarsal Metatarsal Dowel Arthrodesis with Trephine Reamers and Cancellous Bone Plugs: A Technique Guide and Case Report. J Arthritis 5: 198. doi:10.4172/2167-7921.1000198

Page 2 of 5

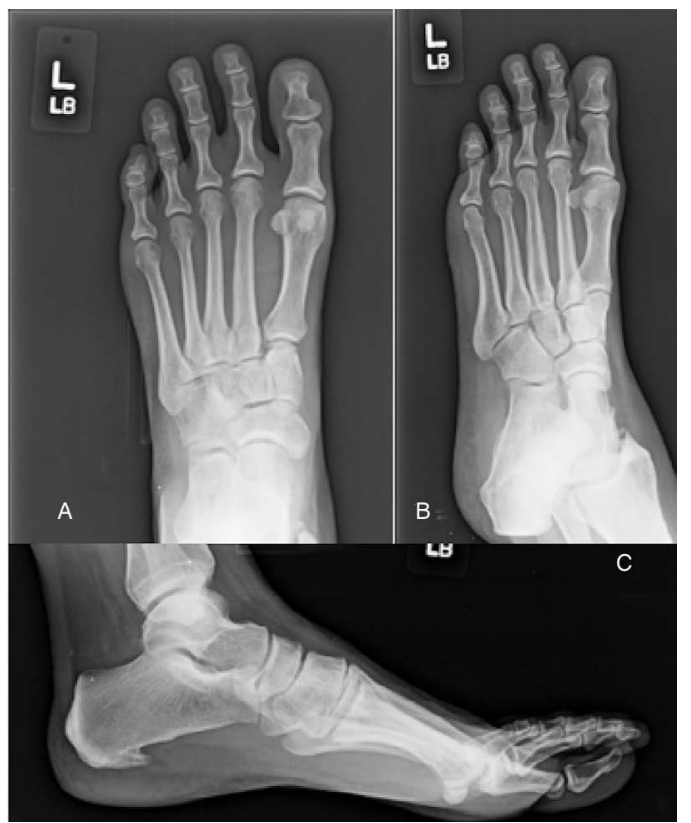

Figure 1: Preoperative radiographs of the (a) AP and (b) Mortise views demonstrating adequate joint space and no obvious degenerative joint disease. (c) Lateral view demonstrates a mild dorsal exostosis (arrow) at the second TMT joint, but otherwise normal.

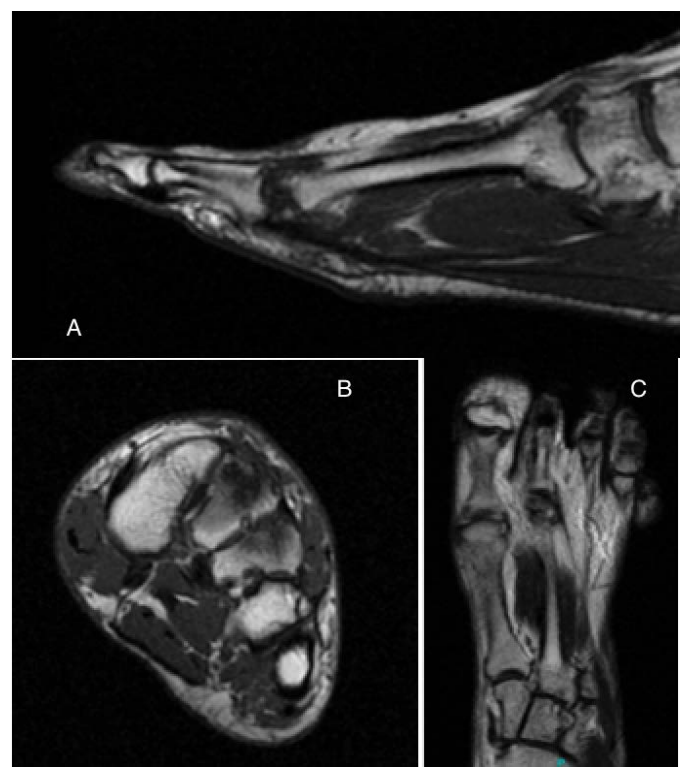

Figure 2: Left foot MRI T1 (a) Sagittal (b) Frontal (c) Axial images demonstrate degenerative changes at the central (second and third) TMT joints with subchondral cyst formation and subtle chonrdal breakdown.

of subchondral cystic formation, osteophytes, chondralmalacia, and subtle bursitis. An injection was provided to the central TMT joint capsule and provided both diagnostic and therapeutic effects for the patient and short-term symptomatic relief. After further conservative management including limited weight bearing in a Controlled-Ankle Motion (CAM) boot and physical therapy failed to provide lasting benefits, the patient consented to arthrodesis of her central (second and third) TMT joints.
The patient was brought to the operating suite and placed on the operating table in the supine position. An ipsilateral hip bump was utilized to rotate the patient out of external rotation for optimal approach. A well-padded calf tourniquet was utilized for hemostasis. After exanguinating the left lower extremity the tourniquet was inflated to $250 \mathrm{mmHg}$. A $5 \mathrm{~cm}$ linear incision was made between the patients second and third TMT joints. Care was taken to lateralize and protect the intermediate dorsal cutaneous nerve and extensor hallucis brevis tendon. A \#15 blade was further utilized to extend the margins of her central (second and third) TMT joints (Figure 3). Placement of the dorsal compression claw plate (Wright Medical Technology, Memphis TN) was performed initially to make sure no interference would occur, at the second and third TMT joint respectively, after placement of the dowel allograft plug (Community Tissue Services, Dayton $\mathrm{OH}$ ).

Fluoroscopy confirmed central placement of a dorsal compression claw plate (Wright Medical Technology, Memphis TN) where $20 \mathrm{~mm}$ screws were inserted at a distance not to interfere with the TMT joints (Figure 4). The hardware was subsequently removed so a guidewire (Arthrex Bio-Tenodesis ${ }^{\mathrm{TM}}$ Instrument Tray, Naples FL) could be inserted directly in the center of the second TMT joint (Figure 5a). The preoperative radiographic width of the joint was $11 \mathrm{~mm}$. An $8.5 \times 18$ $\mathrm{mm}$ dowel shaped allograft plug (Community Tissue Services, Dayton

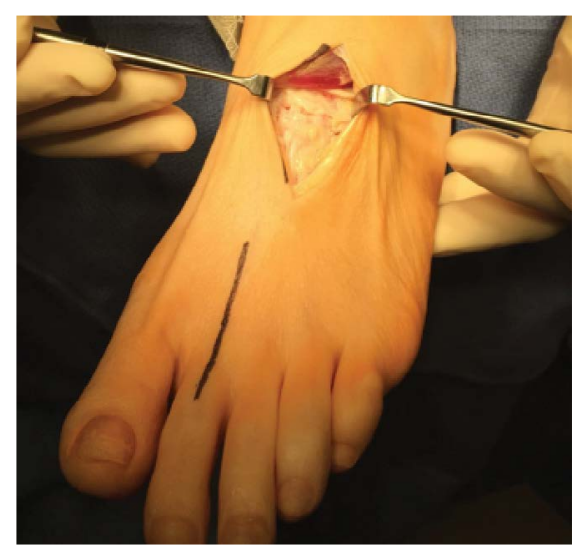

Figure 3: Dorsal view showing degenerative joint disease with chondral wear of the central (second and third) TMT joints after soft tissue dissection.

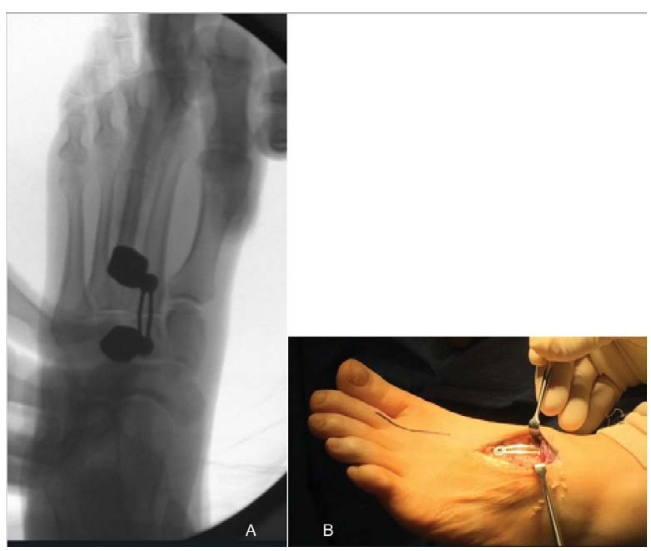

Figure 4: (a) Fluoroscopic guidance for the placement of the dorsal claw plate (Wright Medical Technologies Inc., Memphis TN) prior to joint resection. (b) Shows a clinical comparison demonstrating optimal placement of the claw plate (Wright Medical Technologies Inc., Memphis TN). 
Citation: Downey MW, Fleming JJ, Elgamil B (2016) In Situ Tarsal Metatarsal Dowel Arthrodesis with Trephine Reamers and Cancellous Bone Plugs: A Technique Guide and Case Report. J Arthritis 5: 198. doi:10.4172/2167-7921.1000198

Page 3 of 5

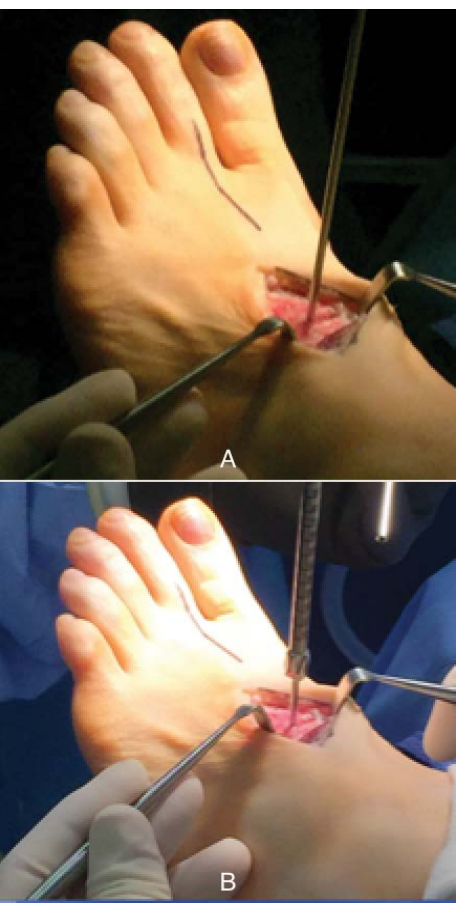

Figure 5: (a) Demonstrates the insertion of the guidewire and (b) shows the subsequent reaming with the $8.5 \mathrm{~mm}$ trephine reamer (Arthrex BioTenodesis $^{\mathrm{TM}}$ Instrument Tray, Naples FL) of the second metatarsocuneiform joint.

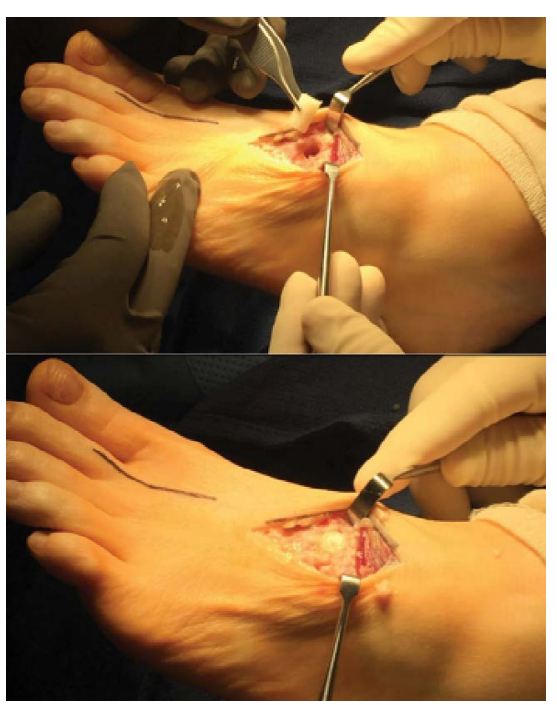

Figure 6: Demonstrates the insertion of the $8.5 \times 18 \mathrm{~mm}$ dowel allograft plug (Community Tissue Services, Dayton $\mathrm{OH}$ ) press fit into the $8.5 \mathrm{~mm}$ reamed dowel site.

$\mathrm{OH})$ was selected in order to preserve the medial and lateral flares of the joint around the allograft. Care was taken to remove any cartilage that may be part of the allograft plug. The selection of the dowel autograft determined our direct size selection the reamer. Under cool lavage (saline irrigation), a $8.5 \mathrm{~mm}$ cannulated trephine reamer (Arthrex Bio- Tenodesis ${ }^{\text {na }}$ Instrument Tray, Naples FL) was utilized to resect the second TMT joint, leaving a small amount of the plantar cortex intact (Figure 5b). Due to mild invagination of dorsal ligamentous tissue, a $4 \mathrm{~mm}$ round burr and rongeur were further utilized to clean around the dowel site. The dowel allograft plug (Community Tissue Services, Dayton $\mathrm{OH}$ ) was aligned into the hole and press fit in until flush dorsally (Figure 6). The dorsal compression claw plate (Wright Medical Technology, Memphis TN) was re-applied, fixated, and the manual tensioner was then utilized to obtain compression (Figure 7). The same procedure as the second TMT joint was then performed for the third TMT joint.

The subcutaneous and skin closure was performed with absorbable and non-absorbable suture respectively. The patient was placed into a non-weight bearing short leg splint and seen in clinic two weeks after her index procedure for suture removal. Radiographs were obtained (Figure 8) and the patient was transitioned into a controlled ankle motion CAM boot for four weeks. The patient was seen at 6 weeks and progressive weight bearing $(40 \mathrm{lbs})$ was initiated in the CAM boot for 2 weeks due to consolidation at the dowel arthrodesis sites and minimal pain over the incision. Physical therapy was also initiated for strength and function. At 8 weeks the patient was transitioned to full weight bearing in the CAM boot as she was pain free with the progressive weight bearing protocol. At 10 weeks the patient was healed radiographically and clinically then transitioned to regular shoes and low impact exercises (Figure 9). At her one year follow up she had no complaints of pain and has returned to all exercises and activities without any restrictions. The patients' radiographs demonstrate a consolidated midfoot fusion without hardware failure (Figure 10).

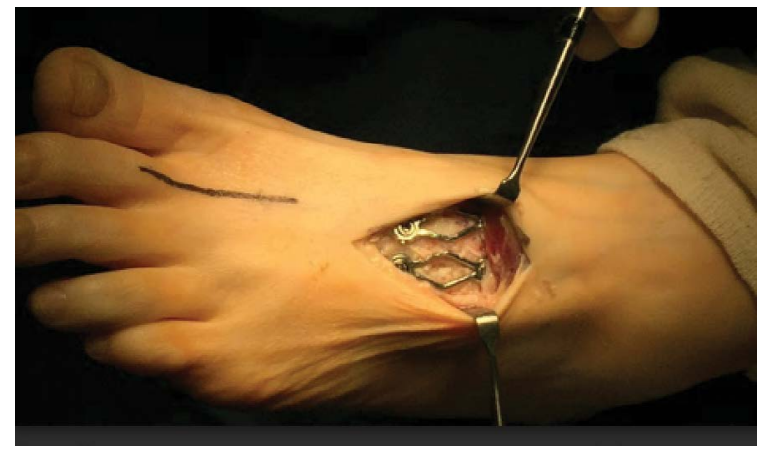

Figure 7: Demonstrates a dorsal view after fixation and subtle compression with the claw plate (Wright Medical Technology Inc., Memphis TN) applied over the dowel allograft plugs (Community Tissue Services, Dayton $\mathrm{OH}$ ).

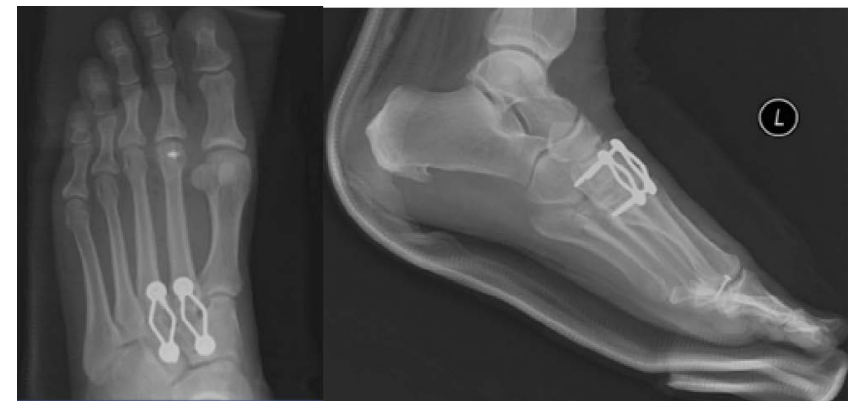

Figure 8: Shows the post-operative radiographs and confirms the in situ dowel allograft plug (Community Tissue Services, Dayton $\mathrm{OH}$ ) placement into the central (second and third) TMT joints with adequate compression and fixation from the claw plate (Wright Medical Technology Inc., Memphis TN). 


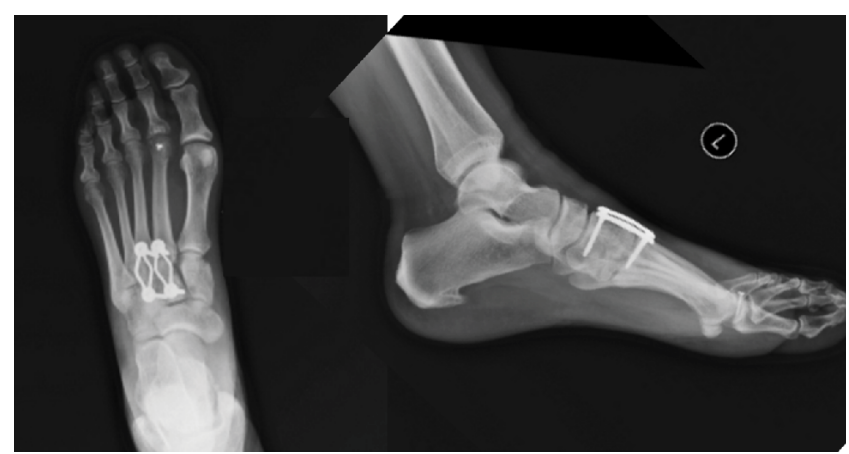

Figure 9: Demonstrates the post-operative radiographs at 6 weeks with early consolidation and graft healing.

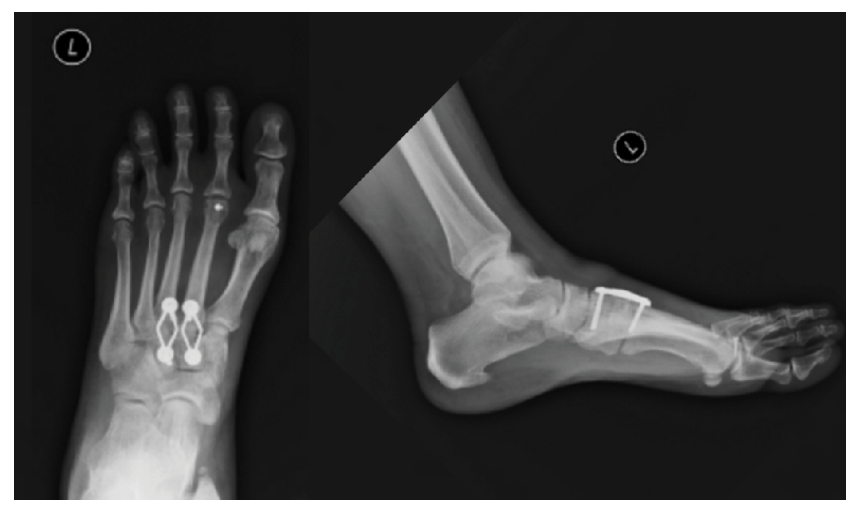

Figure 10: Shows radiographic healing 1-year post operatively with complete consolidation of the in situ dowel allograft plug (Community Tissue Services, Dayton $\mathrm{OH}$ ) and no signs of hardware failure.

\section{Discussion}

Pathology at the TMT joints play a role in continued foot fatigue and further compensation of gait laterally to avoid discomfort over the medial and central columns of the midfoot. Sequeula and further pathology to the central TMT joints are multifactorial. Predisposing risk factors for midfoot arthrosis have been linked to obesity, female gender, gastrocnemius equinus and a long second metatarsal [10]. Direct force to the dorsum of the foot or indirect longitudinal force can be attributed to degenerative disease of this stable complex [11]. Motor vehicle accidents, industrial accidents, falls, and athletic activities make up a large majority of injuries to the midfoot, whereas $60-70 \%$ of degenerative arthritis and cartilage damage is related to later complications of the initial traumatic injury [12]. Impact loading and repetitive stress will continue to biomechanically affect the articular cartilage [13]. Continued degenerative mechanisms can develop when unmatched hardness/stiffness occurs between articular surfaces leading to abrasive wear [14]. Liu et al. [11] found that no variations in biomechanical properties and thickness exist between the second metatarsal and intercunieform articular surfaces. The authors conclude that the well-matched articular surfaces are not predisposed to degenerative changes [11]. Atraumatic TMT joint changes have been linked to pes planovalgus, hallux valgus, and rockerbottom (Charcot) deformities [15]. These are beyond the scope of this in situ TMT joint arthrodesis technique guide and involve deformity modification with corrective procedures throughout the foot and ankle.

Various techniques have been described for recalcitrant midfoot arthrosis of the TMT joints to include cheilectomy, arthroplasty, and arthrodesis. Cheilectomy or exostectomy are viable options for patients with dorsal exostosis and painful prominence independent of deep joint pain [16]. Resection arthroplasty has been described by Gilheany and Amir [17] for older or obese patients with comorbidities that increase the risk of nonunion and/or cannot tolerate prolonged non weight bearing. Even though the authors describe a feasible technique for these high-risk patients, to resect the central TMT joints would render a relatively stable complex unstable. Furthermore, this would alter he foot both biomechanically and anatomically, leading to additional insufficiency and a more challenging reconstructive procedure should it be needed at a later date. Surgical intervention with midfoot arthrodesis continues to be the gold standard for TMT joint pathology. Several authors have described TMT joint arthrodesis without the need for bone graft; however there have been reports of greater nonunion risks when bone graft is not utilized [2] Withey et al. [16] describes a similar trephine dowel arthrodesis with calcaneal autogenous bone graft [16]. They prefer autogenous bone graft as opposed to bone substitutes for osteoconduction, osteoinduction, and osteogenesis properties [16]. The authors report the practicality of this technique and have not come across any nonunions (0\%) [16]. Johnson et al. [18] performed a histological evaluation of cadaveric joints in the foot and found that a layer of calcified cartilage remains when utilizing curettage as opposed to blade resection [18]. This could lead to possible nonunion and/or delayed consolidation of the arthrodesis site. Withey et al. [16] relate that the trephine preparation for arthrodesis include the subchondral plates to reduce the risk of nonunion [16]. Losing the small amount of motion available at the TMT joints will not have impact on function as the trephine dowel arthrodesis technique provides removal of painful micro motion at an already stiff pathologic joint. Furthermore, this limits the risk of iatrogenic transfer metatarsalgia as well as maintains anatomic length [16].

Autogenous bone graft harvested from the iliac crest, the proximal tibia, the distal tibia, and the calcaneus have been described for augmentation [19]. Hyer et al. [20] have reported a higher amount of osteoblastic progenitor cells when performing bone marrow aspirate at different anatomic locations between the anterior iliac crest, the distal tibia, and the calcaneus [20]. The iliac crest is a more favored site biologically and more robust statistically even in the presence of comorbidities to include tobacco use and diabetes [20]. Despite the significant different of osteoblastic progenitor cells the donor site morbidity continues to be problematic and is associated with several complications to include protracted pain, delayed healing, numbness/ neuritis, hematoma, infection, and visceral injury [21,22] Other authors have described simple techniques for trephine calcaneal bone graft $[16,23]$. These offer the advantage of autogenous graft but they are not without postoperative complications, to include sural nerve injury and non-healing surgical wound over the lateral calcaneus. Feeney et al. [24] reported consequential risk of calcaneal fracture with their innovated calcaneal tricortical bone graft technique [24]. Though the possibility of calcaneal fracture is reduced with trephine grafting, compared to tricortical bone graft, there is still plausible morbidity with this technique. Daigre et al. [25] looked at postoperative pain levels associated with bone marrow aspirate (BMA) from the iliac crest, the distal tibia, and the calcaneus [25]. The authors found no difference between the distal tibia and the iliac crest; however, at 12 weeks the visual analogue scale (VAS) pain scores were significantly higher in the calcaneus [25]. Though the study focused on BMA with an 11-gauge jamshidi needle and not a trephine, this further demonstrates donor site complications. Filiatrault and Banks [26] experienced successful arthrodesis in 15/16 patients utilizing allogenic freeze-dried bone 
graft for various trephine techniques across the foot and ankle [3]. The authors relate that this is a viable option for the trephine arthrodesis due to the high rate of fusion and the addition of no donor site morbidity [26]. Cizek et al. [27] relate difficulty assessing consolidation and bridging across the fusion site due to fixation overlap and partial joint margin preservation [27]. Fixation selection is surgeon dependent. There have been numerous fixation combinations described for TMT joint arthrodesis to include lag screws, static screws, neutralization plates, compression plates, as well as staples [28]. Even though the cancellous plug can be press fit into position the authors recommend low profile plates with or without locking options. The trephine dowel graft technique has been successfully reported in other joints in the foot and ankle; however, the authors deduce that the central TMT joints are easier anatomical areas for the trephine dowel arthrodesis [26].

\section{Conclusion}

In conclusion the dowel arthrodesis with the trephine reamers at the central TMT joints and further augmentation with cancellous bone plugs have provided successful treatment of midfoot arthritis. This technique guide is not without its inherent limitations. Larger retrospective or prospective studies are needed to provide more widespread result of the technique's success. This simple, straightforward procedure reduces soft tissue dissection, eliminates arthritic pain, prevents donor graft morbidity, and allows anatomical maintenance without disrupting adjacent anatomical components of the midfoot.

\section{Informed Consent}

Informed consent has been obtained from the patient and/ or appropriate persons for publication, including any necessary photographs.

\section{Conflict of Interest}

Justin J. Fleming, DPM, FACFAS2 is a paid consultant for Arthrex Inc. Naples, FL, USA and Stryker Inc., Kalamazoo, MI, USA. The above companies for the case report provided no funding or direction for the article preparation and submission. Other authors certify that they have no commercial associations (eg, consultancies, stock ownership, equity interest, patent/licensing arrangements, etc) that might pose a conflict of interest in connection with the submitted article.

\section{References}

1. Den Hartog BD, Kay DB (2009) Non-neuropathic midfoot multiplanar deformity: surgical strategies for reconstruction. Foot Ankle Clin 14: 383-392.

2. Sangeorzan BJ, Veith RG, Hansen ST Jr (1990) Salvage of Lisfranc's tarsometatarsal joint by arthrodesis. Foot ankle 10: 193-200.

3. DiDomenico LA, Cross D (2012) Tarsometatarsal/Lisfranc joint. Clin Podiatr Med Surg 29: 221-242.

4. de Palma L, Santucci A, Sabetta SP, Rapali S (1997) Anatomy of the Lisfranc joint complex. Foot Ankle Int 18: 356-364.

5. Peicha G, Labovitz J, Seibert FJ, Grechenig W, Weiglein A, et al. (2002) The anatomy of the joint as a risk factor for Lisfranc dislocation and fracturedislocation. An anatomical and radiological case control study. J Bone Joint Surg $\mathrm{Br}$ 84: 981-985

6. Myerson MS (1999) The diagnosis and treatment of injury to the tarsometatarsal joint complex. J Bone Joint Surg Br 81: 756-763.
7. van der Werf GJ, Tonino AJ (1984) Tarsometatarsal fracture-dislocation. Acta Orthop Scand 55: 647-651.

8. Ryan JD, Timpano ED, Brosky TA $2^{\text {nd }}(2012)$ Average depth of tarsometatarsa joint for trephine arthrodesis. J Foot Ankle Surg 51: 168-171.

9. Philbin T, Rosenberg G, Sferra JJ (2003) Complications of missed or untreated Lisfranc injuries. Foot Ankle Clin 8: 61-71.

10. Davitt JS, Kadel N, Sangeorzan BJ, Hansen ST Jr, Holt SK, et al. (2005) An association between functional second metatarsal length and midfoot arthrosis. J Bone Joint Surg Am 87: 795-800.

11. Liu GT, Lavery LA, Schenck RC Jr, Lanctot DR, Zhu CF, et al. (1997) Human articular cartilage biomechanics of the second metatarsal intermediate cuneiform joint. J Foot Ankle Surg 36: 367-374.

12. Hardcastle PH, Reschauer R, Kutscha-Lissberg E, Schoffmann W (1982) Injuries to the tarsometatarsal joint. Incidence, classification and treatment. Bone Joint Surg Br 64: 349-356.

13. Radin EL, Paul IL (1971) Response of joints to impact loading. I. In vitro wear Arthritis Rheum 14: 356-362.

14. Armstrong CG, Mow VC (1982) Variations in the intrinsic mechanical properties of human articular cartilage with age, degeneration, and water content. J Bone Joint Surg Am 64: 88-94.

15. Jung HG, Myerson MS, Schon LC (2007) Spectrum of operative treatments and clinical outcomes for atraumatic osteoarthritis of the tarsometatarsal joints. Foot Ankle Int 28: 482-489.

16. Withey CJ, Murphy AL, Horner R (2014) Tarsometatarsal joint arthrodesis with trephine joint resection and dowel calcaneal bone graft. J Foot Ankle Surg 53: 243-247.

17. Gilheany MF, Amir OT (2013) Metatarsocuneiform joint resection arthroplasty for atraumatic osteoarthrosis: an alternative to arthrodesis. J Foot Ankle Surg 52: $122-124$

18. Johnson JT, Schuberth JM, Thornton SD, Christensen JC (2009) Joint curettage arthrodesis technique in the foot: a histological analysis. J Foot Ankle Surg 48: 558-564.

19. O'Keeffe RM Jr, Riemer BL, Butterfield SL (1991) Harvesting of autogenous cancellous bone graft from the proximal tibial metaphysis. A review of 230 cases. J Orthop Trauma 5: 469-474.

20. Hyer CF, Berlet GC, Bussewitz BW, Hankins T, Ziegler HL, et al. (2013) Quantitative assessment of the yield of osteoblastic connective tissue progenitors in bone marrow aspirate from the iliac crest, tibia, and calcaneus. J Bone Joint Surg Am 95: 1312-1316.

21. Schon LC, Acevedo JI, Mann MR (1999) Sliding wedge local bone graft for midfoot arthrodesis. Foot Ankle Int 20: 340-341.

22. DeOrio JK, Farber DC (2005) Morbidity associated with anterior iliac crest bone grafting in foot and ankle surgery. Foot Ankle Int 26: 147-151.

23. Roukis T (2006) A simple technique for harvesting autogenous bone grafts from the calcaneus. Foot Ankle Int 27: 998-999.

24. Feeney S, Rees S, Tagoe M (2007) Tricortical calcaneal bone graft and management of the donor site. J Foot Ankle Surg 46: 80-85.

25. Daigre JL, DeMill SL, Hyer CF (2015) Assessment of Bone Marrow Aspiration Site Pain in Foot and Ankle Surgery. Foot Ankle Spec

26. Filiatrault AD, Banks AS (2006) Trephine arthrodesis of the foot and ankle: indications, operative technique, and long-term follow-up. J Am Podiatr Med Assoc 96: 198-204.

27. Cizek GR, Boyd LM (2000) Imaging pitfalls of interbody spinal implants. Spine (Phila Pa 1976) 25: 2633-2636.

28. Smith SE, Camasta CA, Cass AD (2009) A technique for isolated arthrodesis of the second etatarsocuneiform joint. J Foot Ankle Surg 48: 606-611. 